\title{
Reflectancia solar de las envolventes opacas de la ciudad y su efecto sobre las temperaturas urbanas
}

\section{Solar reflectance of opaque enveloped and its effect on urban temperatures}

\author{
$\underline{\text { N. Alchapar }}^{(*)}$, E. Correa ${ }^{(*)}$
}

\section{RESUMEN}

La selección adecuada de las envolventes contribuye a disminuir las temperaturas de la ciudad. En este trabajo se caracterizó el comportamiento térmico de 150 materiales disponibles a nivel local mediante el Índice de Reflectancia Solar (SRI) y se determinó su impacto sobre la temperatura urbana. Para ello se simularon 6 escenarios en la ciudad de MendozaArgentina, mediante el uso del software ENVI-met. Los resultados revelaron que aunque el color es una variable determinante del desempeño térmico superficial de un material su comportamiento puede ser mejorado o empobrecido por el efecto de otras características como: terminación, composición y envejecimiento. A igualdad de color, estas características pueden modificar la temperatura superficial de un material entre 20 y $29{ }^{\circ} \mathrm{C}$. A escala urbana, los resultados muestran que la selección adecuada de materiales de la envolvente urbana podría disminuir hasta $3{ }^{\circ} \mathrm{C}$ la temperatura del aire y $12{ }^{\circ} \mathrm{C}$ la temperatura de las superficies.

Palabras clave: Microclima; envolvente urbana; comportamiento térmico; características de los materiales.

\section{ABSTRACT}

In order to contribute to the proper selection of envelopes to reduce the city temperature, we characterized the thermal behavior of 150 materials available locally, by Solar Reflectance Index. With the purpose to determine the impact of the reflectance of the materials on an urban scale, six urban scenarios were simulated in the city of Mendoza-Argentina, using the software ENVI-met. The results revealed that although the color is a determining variable of the surface thermal behavior of a material, it can be improved or impoverished by the effect of other features such as termination and composition up to $2 \mathrm{O}^{\circ} \mathrm{C}$; as well as by the aging up to $29^{\circ} \mathrm{C}$. At the urban level, the results show that the proper selection of materials of the urban envelope could be reduced up to $3{ }^{\circ} \mathrm{C}$ the air temperature and up to $12{ }^{\circ} \mathrm{C}$ the surface temperature.

Keywords: Microclimate; urban envelope; thermal behavior; materials characteristics.

${ }^{(*)}$ Instituto Ciencias Humanas Sociales y Ambientales, CCT-Mendoza, CONICET. Argentina.

Persona de contacto/Corresponding author: nalchapar@mendoza-conicet.gob.ar (N. Alchapar)

Cómo citar este artículo/Citation: Alchapar, N., Correa, E. (2015). Reflectancia solar de las envolventes opacas de la ciudad y su efecto sobre las temperaturas urbanas. Informes de la Construcción, 67(540): e112, doi: http://dx.doi.org/10.3989/ic.14.131.

Licencia / License: Salvo indicación contraria, todos los contenidos de la edición electrónica de Informes de la Construcción se distribuyen bajo una licencia de uso y distribución Creative Commons Reconocimiento no Comercial 3.o. España (cc-by-nc). 


\section{INTRODUCCIÓN}

Las áreas urbanas cubren sólo el 2,8 \% del total de nuestro planeta, sin embargo, son responsables del $75 \%$ del consumo mundial de recursos (1) (2). De acuerdo con el Key World Energy Statistic 2013 de la Agencia Internacional de Energía (3), el consumo de energía global anual ha crecido de 4000 MTEP (millones de toneladas equivalentes de petróleo) a casi 9000 MTEP durante los últimos 40 años (1971 a 2011), durante el mismo período, las emisiones de $\mathrm{CO}_{2}$ se han duplicado. La intensificación de consumo energético no sólo provoca dificultades de abastecimiento y agotamiento de los recursos energéticos fósiles, sino que también influye de manera significativa sobre las condiciones de la vida humana. Provocando una debilitación del medio ambiente mundial, como consecuencia de la afectación de la capa de ozono, el calentamiento global, los cambios climáticos, entre otros fenómenos (4). Sumado a estas tendencias alarmantes, el $32 \%$ del consumo total de energía final y casi el $40 \%$ del consumo de energía primaria son atribuibles a los edificios.

La urbanización produce la alteración antropogénica del clima en el que se implanta una ciudad, y genera un efecto conocido como isla de calor urbana (ICU). La ICU afecta, tanto de manera directa como indirecta, el consumo de energía, la habitabilidad y la calidad del aire de las ciudades (4) (5) (6) (7) (8) (9) (10) (11).

De modo particular, la superficie de la ciudad se compone de un mosaico de diferentes materiales, a los que denominamos la envolvente urbana, cada uno de ellos muestran distintos comportamientos energéticos en función de sus propiedades térmicas y ópticas, lo que impacta en forma directa dentro del microclima local. Cada material que compone la envolvente urbana tiene diferente capacidad de reflejar la radiación solar recibida (reflectancia solar o albedo) lo cual altera la fracción de radiación solar absorbida por la ciudad. La mayoría de los materiales de construcción tienen baja reflectancia solar en comparación con los materiales naturales. Esta combinación de baja reflectancia solar y alta capacidad calorífica difícilmente ocurre en la naturaleza. Debido a que los materiales que componen las envolventes urbanas son responsables de la interacción entre el edificio y el medio ambiente, afectan tanto el consumo de energía para el acondicionamiento térmico de los edificios como las temperaturas de la ciudad. Todo esto, avala como hipótesis que intervenir positivamente sobre estos componentes del hábitat contribuye a alcanzar un diseño edilicio y un desarrollo urbano sustentables.

Este estudio muestra los resultados sustanciales de una investigación desarrollada en el marco de la ejecución de dos proyectos de investigación desarrollados en la UID - Laboratorio de Ambiente Humano y Vivienda - Instituto Ciencias Humanas Sociales y Ambientales (INCIHUSA) Consejo Nacional de Investigaciones Científicas y Técnicas (CONICET)-: PIP: 2012-2014 ${ }^{1}$ y PICT 2011- 2015². Los proyectos están vinculados a desarrollar estrategias de mitigación del efecto de isla de calor urbana para contribuir a la sustentabilidad urbano-edilicia y mejorar la calidad del hábitat humano.
El objetivo final es contribuir a la selección de los materiales más adecuados para disminuir las temperaturas urbanas y de ese modo combatir la isla de calor, mejorar la habitabilidad de los espacios públicos y disminuir el consumo energético de los edificios.

\section{MATERIALES Y MÉTODOS}

El abordaje metodológico de esta investigación comprende dos escalas de intervención: escala objeto (Sección 2.1 y 2.2) y escala urbana (Sección 2.3 y 2.4).

A escala objeto, se desarrollan experimentos bajo condiciones controladas de materiales opacos disponibles en el mercado local, con el fin de caracterizar su comportamiento óptico y temperatura superficial bajo condiciones de material nuevo y envejecido. Para caracterizar estos materiales se utilizó el Índice de Reflectancia Solar o SRI por sus siglas en inglés. Establecido por la norma ASTM E1980-11: Standard Practice for Calculating Solar Reflectance Index of Horizontal and Low-Slopped Opaque Superface (12).

A escala urbana, se evalúa mediante simulaciones, el efecto de la aplicación de materiales con distintas características sobre las envolventes y su impacto sobre las temperaturas del aire y del suelo. Se ajustó el modelo numérico con los resultados del monitoreo de un espacio real y se simularon sobre este, distintos escenarios que combinan densidades edilicias y rangos de albedo de la envolvente. Las simulaciones se llevaron a cabo mediante el software ENVI-met 3.1 desarrollado por Bruse, M. (1999) en el Instituto de Geografía de la Universidad de Mainz - Alemania (13). El uso de esta herramienta está ampliamente validado a nivel internacional (14) (15) (16) (17) (18) (19) (20).

ENVI-Met 3.1 es un modelo computacional tridimensional que trabaja a escala de microclima urbano, diseñado para simular las interacciones entre el aire y la superficie del entorno urbano con una resolución típica de 0,5 a $10 \mathrm{~m}$ en el espacio y cada 10 segundos en tiempo. Es un modelo de pronóstico basado en las leyes fundamentales de la dinámica de fluidos y la termodinámica. Incluye la simulación de flujos alrededor y entre edificios; procesos de intercambio de calor y vapor de las superficies de suelo y paredes; turbulencia; parámetros de la vegetación; bioclimatología; y dispersión de contaminantes (21). Se puede obtener información sobre el programa, así como de las versiones actualizadas en: http://www.envi-met. com/documents/onlinehelpv3/ helpindex.htm.

\subsection{Ensayo y caracterización de materiales}

De acuerdo a su disponibilidad en el mercado local se seleccionaron 150 materiales. Dentro de los materiales de disposición horizontal (pisos y techos) se analizaron: 38 pavimentos peatonales (cementicios y graníticos) y 16 tipologías de tejas (cerámicas y cementicias). Dentro de los materiales de disposición vertical se estudiaron 80 revestimientos texturados (acrílicos y cementicios) y 16 pinturas látex para exterior

\footnotetext{
Proyecto investigación plurianual - CONICET: «Eficiencia térmica, económica y ambiental de los materiales utilizados en la envolvente de los espacios abiertos y valoración de su eficacia como estrategia de mitigación de la isla de calor en ciudades de zonas áridas». Titular del proyecto: Dra. Erica Correa.

2 Proyecto de investigación de ciencia y tecnología - CONICET: «Diseño eficiente y nuevas tecnologías verdes para la consecución de la...». Titular del Proyecto: M. Alicia Cantón.
} 
(acrílicas, impermeables y atérmicas). Los mismos fueron dispuestos sobre una superficie horizontal de poliestireno expandido que actúa como barrera adiabática de la conductividad del material a la tierra.

Para sistematizar el análisis de los resultados los materiales fueron clasificados de acuerdo a su composición, terminación/textura, color y forma, según detalle en Tabla 1.

\subsection{Propiedades ópticas e Índice de Reflectancia Solar de materiales}

Para la evaluación de las propiedades ópticas y la determinación del SRI de cada material, los mismos se dispusieron sobre una superficie horizontal de poliestireno expandido de 10 $\mathrm{cm}$ de espesor. Las variables medidas fueron emisividad $(\varepsilon)$ con una termocupla tipo $\mathrm{T}$ asociado a un data logger Hobo U12, según norma ASTM E1993-99ª/o6 (22); reflectancia solar (â) con un albedómetro tipo Kipp \& Zonen CMA11, según método variante de la norma ASTM E1918-97/o6 (23); temperatura superficial (Ts) con una cámara infrarroja tipo Fluke Ti 55; y radiación solar sobre superficie horizontal (I) y temperatura del aire (Ta), registradas con una estación meteorológica móvil tipo $\mathrm{HOBO} 囚(\mathrm{H} 21-001)$.

La combinación de estas variables con respecto a una superficie patrón de color negra (o \%) y otra blanca (100 \%), da como resultado un factor que caracteriza el comportamiento térmico del material definido como su Índice de Reflectan- cia Solar (SRI). El método establecido por la norma ASTM E1980-11 para calcular el SRI se utiliza para superficies con emisividades mayores a 0,01 y temperaturas superficiales menores a $150^{\circ} \mathrm{C}$. Para una superficie expuesta al sol y aislada por debajo se obtiene la temperatura superficial de equilibrio (Ts) a partir de la siguiente ecuación [1].

$$
\begin{aligned}
\mathrm{Ts}= & 309,07+\frac{(1066,07 \alpha-31,98 \varepsilon)}{\left(6,78 \varepsilon-\mathrm{h}_{c}\right)}- \\
& -\frac{\left(890,94 \alpha^{2}-2153,8 \alpha \varepsilon\right)}{\left(6,78 \varepsilon+\mathrm{h}_{c}\right)}
\end{aligned}
$$

Por lo tanto, el Índice de Reflectancia Solar se define como el producto del resultado de la sustracción de la temperatura patrón negra menos la temperatura del material testeado, dividido el valor dado por la resta de la temperatura del patrón negro menos la temperatura del patrón blanco [2].

$$
S R I=100 \frac{T_{b}-T_{s}}{T_{b}-T_{w}}
$$

Con el tiempo, el SRI puede ser modificado por efecto del envejecimiento. Es por ello, que durante un período de tres años (2011 a 2013) se monitoreó la reflectancia solar (â), emisividad y temperatura superficial del total de los materiales, al igual que para calcular el SRI inicial (SRI), y se determinaron los valores de SRI para los dos períodos de envejecimiento ( $\mathrm{SRI}_{2}$ y SRI SR. $_{3}$.

\begin{tabular}{|c|c|c|c|c|c|c|}
\hline \multicolumn{2}{|c|}{ CARACTERÍSTICAS } & COMPOSICIÓN & \multicolumn{2}{|c|}{$\begin{array}{l}\text { TERMINACIÓN/ } \\
\text { TEXTURA }\end{array}$} & COLOR & FORMA \\
\hline \multirow{6}{*}{ 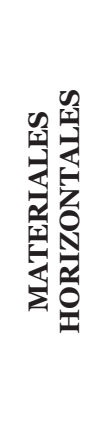 } & \multirow{4}{*}{$\sum_{\substack{\mid \\
\vdots}}^{0}$} & Cementicia & \multicolumn{2}{|l|}{ Rústica } & rojo; amarillo; gris; negro & \multirow{4}{*}{$\begin{array}{l}\text { Recto: cuadrado; dos panes; vainilla; } \\
\text { diagonal } \\
\text { Circular: araña; abanico; Andalucía } \\
\text { Liso: mosaico; estrella; canto rodado }\end{array}$} \\
\hline & & Granítica & \multicolumn{2}{|l|}{ Pulida } & \multirow{2}{*}{$\begin{array}{l}\text { travertino; bordeaux; verde; } \\
\text { negro; gris; multicolor }\end{array}$} & \\
\hline & & Cementicia-pétrea & \multicolumn{2}{|c|}{ Rústica; pulida } & & \\
\hline & & Cementicia-calcárea & \multicolumn{2}{|l|}{ Pulida } & rojo; amarillo; negro & \\
\hline & $\sum_{1}^{2}$ & Cerámica & \multicolumn{2}{|c|}{$\begin{array}{l}\text { Natural; mate; } \\
\text { esmaltada; brillante; } \\
\text { envejecida }\end{array}$} & terracota; negro & \multirow[t]{2}{*}{ Colonial; francesa; romana } \\
\hline & & Cementicia & \multicolumn{2}{|c|}{ Natural; mate } & terracota; gris; negro & \\
\hline \multirow{9}{*}{ 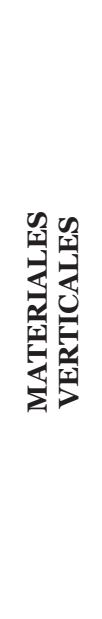 } & & & $\begin{array}{l}\text { Rulato } \\
\text { travertino }\end{array}$ & $\begin{array}{l}\text { fino; } \\
\text { grueso }\end{array}$ & \multirow{6}{*}{$\begin{array}{l}\text { blanco; marfil; piedra parís; } \\
\text { ocre; terracota; gris perla; } \\
\text { verde cemento; gris plomo }\end{array}$} & \\
\hline & ֶֻ & Acrílica & Llaneada & $\begin{array}{l}\text { fino; } \\
\text { grueso }\end{array}$ & & \\
\hline & $\underline{E}$ & & Granitex & medio & & \\
\hline & 易 & \multirow{3}{*}{ Cementicia } & Travertino & $\begin{array}{l}\text { fino; } \\
\text { grueso }\end{array}$ & & \\
\hline & $\underline{\simeq}$ & & Llaneado & $\begin{array}{l}\text { fino; } \\
\text { grueso }\end{array}$ & & \\
\hline & & & Granitex & medio & & \\
\hline & \multirow{3}{*}{$\sum_{i}^{2}$} & Acrílica & \multirow{3}{*}{$\begin{array}{l}\text { Mate; } \\
\text { satinada }\end{array}$} & & \multirow{3}{*}{ blanco; terracota; negro } & \\
\hline & & Impermeable & & & & \\
\hline & & Atérmica & & & & \\
\hline
\end{tabular}

Tabla 1. Clasificación de materiales horizontales (pavimentos peatonales y tejas)

y verticales (revestimientos texturados y pinturas látex de exterior) según composición, terminación, textura, color y forma. 


\subsection{Descripción y ajuste de área simulada}

Para determinar el impacto relativo de la adecuada selección de los materiales como estrategia para la rehabilitación urbano-edilicia, se procedió a simular con el software ENVImet 3.1 un área $(210 \times 210 \mathrm{~m})$ de la ciudad de Mendoza, situado en el centro oeste del Área Metropolitana ( $32^{\circ} 54^{\prime} 47^{\prime \prime S}$, $68^{\circ} 50^{\prime} 46^{\prime \prime} \mathrm{O}$ ), zona muy vegetada, que presenta características de uso residencial y baja densidad: altura edilicia promedio $(\mathrm{H})=6 \mathrm{~m}$ y ancho de canal vial $(\mathrm{W})=18 \mathrm{~m}$. Fue seleccionada este área completamente consolida, porque manifiesta un proceso de densificación sostenida y está inserta en un zona de gran potencialidad para la rehabilitación urbana que permite sentar las bases para un futuro crecimiento vertical. Sumado a que es un caso representativo de las condiciones típicas de la ciudad de Mendoza -el $80 \%$ de la ciudad es de densidad baja, el 70 \% de los canales viales son de 20 metros de ancho y está intensamente forestada, razón por la cual recibe la denominación de ciudad Oasis (24).

Para ajustar el modelo físico de ENVI-met se utilizaron registros de temperatura y humedad de aire medidos en el área urbana evaluada. Para obtener estos datos se empleó una estación fija tipo $\mathrm{HOBO} 囚$ (Ho8-003-02), ubicada en el cañón vial Dr. Lemos a una altura de 2,5 metros. Para el ajuste del modelo teórico se seleccionó como día de estudio el 14 de enero 2010 , temperatura promedio igual a $27^{\circ} \mathrm{C}$, con una amplitud térmica de $15^{\circ} \mathrm{C}$, velocidad de viento de $3 \mathrm{~m} / \mathrm{s}$ a $10 \mathrm{~m}$ de altura y humedad relativa de $28 \%$ (características compatibles con las de un día típico de verano en la ciudad de Mendoza). En orden de validar la curva simulada de ENVImet con el día de referencia medido, se procedió a ajustar los resultados simulados con el del punto fijo ubicado dentro del área de estudio. La curva simulada presenta un ajuste con la curva medida de $\mathrm{R}^{2}=0,98$, error típico $=0,7$ y error estándar de la media $=0,025$.

\subsection{Escenarios simulados}

Para determinar el efecto de la aplicación de materiales con distintas características sobre las envolventes y su impacto sobre las temperaturas se simularon 6 escenarios que combinan tres rangos de albedo para la baja y alta densidad respectivamente. Para realizar este análisis los factores indicados en Tabla 2 fueron evaluados.

\section{RESULTADOS A ESCALA OBJETO}

\subsection{Situación térmica de los materiales presentes en el mercado local}

Una vez determinados bajo norma el albedo y emisividad de cada uno de los materiales que componen el muestrario local de elementos disponibles para la resolución de las envolventes de la ciudad, se confeccionó un gráfico con el objetivo de generar una herramienta que permita visualizar rápidamente el comportamiento óptico de los mismos (Figura 1).

Se localiza cada material en un eje de ordenadas $(\mathrm{x}, \mathrm{y})$ correspondiendo el valor en el eje de abscisas inferior a su reflectancia solar y el valor en el eje de ordenadas derecho a su emitancia IR. Del mismo modo pueden conocerse sus propiedades complementarias leyendo en el eje de abscisas superior su absortancia solar y en el de ordenadas a la izquierda su absortancia IR.

Se observa en la Figura 1 que el conjunto relevado (150 materiales) presenta reflectancias dentro del rango del espectro solar que van desde de 0,20 a 0,93; y emitancias en el rango del espectro IR lejano que van desde 0,70 a 0,98.

Se considera que un material es «absortivo» cuando tiene una reflectancia solar o albedo entre o y o,7 y una emisividad infrarroja (IR) entre 0,85 y 1 ; es «reflectivo» cuando tiene una reflectancia solar entre 0,5 y 1 y una emisividad infrarroja (IR) entre 0,7 a 0,85; es «selectivo caliente» cuando tiene una reflectancia solar hasta 0,5 y una emisividad infrarroja (IR) entre 0,7 a 0,85; y es «selectivo frio» cuando tiene una reflectancia solar entre 0,7 y 1 y una emisividad infrarroja (IR) entre 0,85 y 1 (ver Figura 1). Si ubicamos en el gráfico todos los materiales evaluados, de acuerdo a su comportamiento óptico la mayoría de los que componen la ciudad se

Tabla 2. Rango de albedo de la materialidad en baja y alta densidad edilicia.

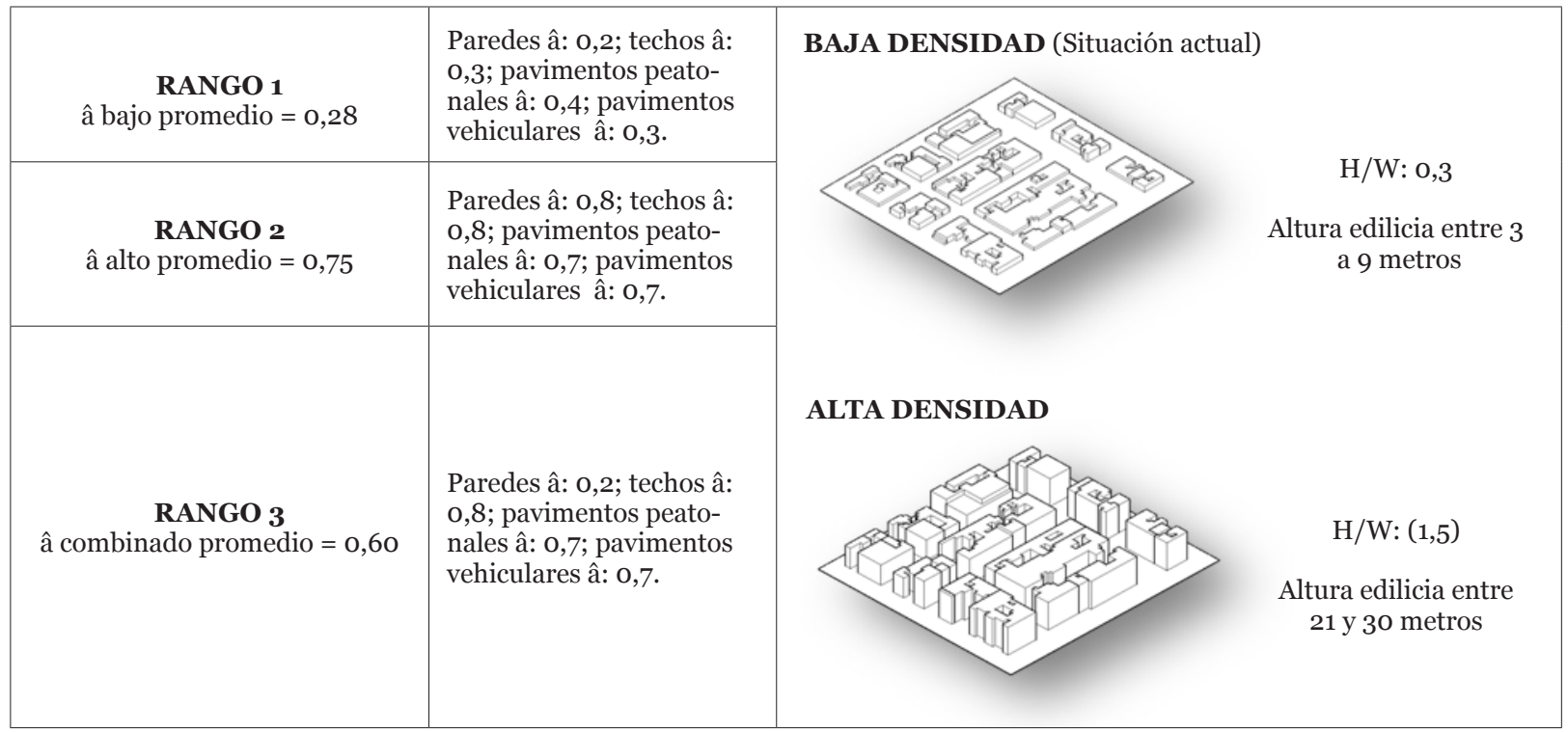


comportan como absortivos, unos pocos como reflectivos, y un grupo acotado responde a las características de los selectivos fríos o también denominados «materiales fríos». Estos en particular constituyen las alternativas que resultan apropiadas para disminuir las temperaturas dentro de una ciudad.

El grupo de «materiales fríos» está constituido en su mayoría por materiales utilizadas para la resolución de superficies verticales, tales como revestimiento texturados en colores blanco, gris perla, marfil, y piedra parís; y algunas pinturas blancas. Dentro de los materiales horizontales clasificados como selectivos fríos se encuentran algunas tejas cerámicas terracota y ninguno de los pavimentos peatonales disponibles en el mercado local. Tan sólo el 13 \% de los materiales de disposición horizontal evaluados se comportan como selectivos fríos, en cambio el $46 \%$ de los materiales verticales alcanzan esta característica.

Cabe destacar, que ninguno de los 38 pavimentos disponibles en el mercado posee características de selectivos fríos y la mayoría corresponde a la categoría materiales absortivos. Si tomamos en cuenta su posición relativa al sol, se evidencia la situación de perjuicio en la que se encuentra la ciudad de Mendoza en lo referente al aumento de cargas térmicas. Sin embargo como hecho positivo y relevante se observa que no hay materiales de la envolvente urbana evaluados que posean características de selectivo calientes (alto reflectancia solar y baja emitancia IR).

A partir de los resultados obtenidos se podría inferir que en términos de intervención, mejorar la reflectancia solar (albedo) de los materiales ofrece mayores posibilidades para reducir las cargas térmicas de las envolventes de la ciudad. Esta información es relevante para la industria a los efectos de decidir hacia dónde direccionar sus esfuerzos para mejorar la certificación energética de sus productos. Especialmente poniendo énfasis en el desarrollo de materiales para la envolvente horizontal (pisos y techos) dado su rol determinante en las ganancias térmicas de un recinto urbano.

\subsection{Selección de los materiales más eficientes de acuerdo a sus características}

Los resultados de los experimentos en condiciones controladas revelaron diferencias de temperatura superficial entre las opciones disponibles en cada grupo muestral. Por ejemplo, en los pavimentos peatonales evaluados las diferencias de temperatura alcanzan los $31^{\circ} \mathrm{C}$, mientras que en tejas son de $22{ }^{\circ} \mathrm{C}$, en revestimientos verticales de $41^{\circ} \mathrm{C}$ y en pinturas exteriores de $39{ }^{\circ} \mathrm{C}$. Estas diferencias responden al efecto combinado de las características particulares del material: color, textura/terminación, composición y forma. Es por ello que con el propósito de analizar los resultados con mayor profundidad, en las siguientes secciones (3.2.1, 3.2.2, 3.2.3, y 3.2.4) se comparan los efectos de la modificación de una de las variables: composición, terminación, color o forma; manteniendo constante las restantes. Por ejemplo, se compara el comportamiento térmico de un pavimento peatonal con igual composición, terminación y color pero diferente forma, y así sucesivamente con las combinatorias posibles de variables intervinientes.

\subsubsection{Pavimentos peatonales}

Se observan las siguientes tendencias:

- La composición no es una condición determinante en el comportamiento térmico de los pavimentos peatonales. Por ejemplo, los pavimentos recto cuadrado gris de com-

\section{PROPIEDADES ÓPTICAS DE MATERIALES}



Figura 1. Propiedades ópticas de materiales presentes en el mercado local y relevado en la ciudad. 
posición cementicia-rústica y cementicia-pétrea registran similitudes en su comportamiento térmico. Ts $=49{ }^{\circ} \mathrm{C} \mathrm{y}$ SRI $=76-77 \%$. (Ver P2O vs. P3o en Tabla 3).

- La terminación pulida en la mayoría de los casos tiene un mejor desempeño térmico que el acabado rústico. Por ejemplo, los pavimentos liso mosaico negro con terminación pulida son $2{ }^{\circ} \mathrm{C}$ más fríos que con terminación rústica. (Ver P19 vs. P29 en Tabla 3)

- Con respecto al color, el mejor comportamiento térmico está vinculado a las tonalidades claras (travertino, gris, rojo $\mathrm{y}$ amarillo) para todas las formas, composiciones y acabado. Estos colores registran temperaturas superficiales entre 30 y $50{ }^{\circ} \mathrm{C}$ (ver P20-25-30-34-36-37-38). En los colores oscuros, por ejemplo el pavimento negro que tiene el mejor desempeño es el Cementicio-pétreo pulido circular andalucía negro multicolor-P26- con una temperatura superficial de $54,5{ }^{\circ} \mathrm{C}(\mathrm{SRI}=66 \%)$. El pavimento menos eficiente es Cementicio rústico circular abanico negro -Po5- con una temperatura superficial de $52{ }^{\circ} \mathrm{C}$ (SRI $52 \%$ ). Es decir que la incorporación de chispas multicolor en el material negro, mejora $2,5{ }^{\circ} \mathrm{C}$ el desempeño respecto a la condición más desfavorable. (Ver P26 vs. Po5 en Tabla 3).

- La forma circular andalucía es las más ineficiente para el caso de los pavimentos cementicios rústicos negros. Es decir que a igual composición, terminación y color, se registraron incrementos de temperatura superficial entre 1 a $3{ }^{\circ} \mathrm{C}$ superiores en la alternativa circular andalucía -Po5. Para un mismo color (negro), terminación (rústico) y composición (cementicia), la forma recta cuadrada -P33- es la más eficiente. El valor del SRI entre alternativas evaluadas oscila entre 58 \% y $59 \%$. (Ver Po5 vs. P33 en Tabla 3).

\subsubsection{Tejas}

- Como tendencia general se evidencia que la composición cerámica es mejor que la cementicia. En particular, las composiciones cerámicas de color terracota poseen un comportamiento superior a la misma alternativa de composición cementicia. Por ejemplo, la teja Cerámica natural colonial terracota -To1- muestra una temperatura superficial de $43{ }^{\circ} \mathrm{C}(\mathrm{SRI}=90 \%)$, mientras que la Cementicia natural colonial terracota -T11- tiene una temperatura superficial de $55{ }^{\circ} \mathrm{C}(\mathrm{SRI}=64 \%)$. El mismo fenómeno se observa sobre las tejas de colores oscuros: la composición cerámica tiene una mayor capacidad para disminuir las temperaturas de la superficie de la ciudad, en comparación con la composición cementicia. La temperatura superficial promedio de las tejas cerámicas negras es de $54{ }^{\circ} \mathrm{C}$, mientras que de las cementicias es de $58^{\circ} \mathrm{C}$. (Ver To1 vs. T11 en Tabla 3).

- La terminación esmaltada y natural son térmicamente más eficientes que la mate y envejecido. Por ejemplo en las tejas cerámicas romanas terracota se registraron diferencias de temperatura superficial de hasta $9{ }^{\circ} \mathrm{C}$ más fría para el caso esmaltado (ver To8, To9, T10). En las tejas de composición cementicia francesa negra, la terminación acrílica resulta más eficiente que la mate. Se observaron diferencias térmicas de $3{ }^{\circ} \mathrm{C}$ menos en la tipología acrílica. (Ver T12, T13 en Tabla 3).

- Los colores terracota y gris son más eficientes que el negro. El promedio de temperatura superficial de las tejas color negro es de $58{ }^{\circ} \mathrm{C}$, mientras que las terracotas consiguen un Ts promedio $=47^{\circ} \mathrm{C}$ y un SRI $=22 \%$. Como caso excepcional, se puede ver que entre las tejas terracota, la Cementicia mate francesa terracota -T16- es la más ineficiente ( Ts $=56^{\circ} \mathrm{C}$; SRI $\left.=63 \%\right)$. Eleva su temperatura superficial por sobre dos tejas de color negro: Cerámica brillante bicocción francesa negra -To4-y Cementicia mate colonial negra -T15-. El color gris, en las tejas cementicias -T14- registra la menor temperatura superficial, $\mathrm{Ts}=46^{\circ} \mathrm{C}$ y SRI $=82 \%$.

- La forma colonial muestra el mejor desempeño en términos de temperatura superficial y SRI. Por ejemplo la teja cementicia mate negra de forma francesa -T12- tiene una temperatura superficial igual a $64{ }^{\circ} \mathrm{C}$ y SRI $=47 \%$, mientras que la misma teja de forma colonial -T15- registra una temperatura de $56{ }^{\circ} \mathrm{C}$ y SRI $=63 \%$. (Ver T12-T15 en Tabla 3).

\subsubsection{Revestimientos texturados}

Se observan las siguientes tendencias:

- Con respecto a la composición, se evidencia que la alternativa acrílica tiene mayor capacidad reflectiva y por lo tanto son las opciones que se mantienen más frías. Debido a que el $43 \%$ de los revestimientos acrílicos (SIP) y el $28 \%$ de los revestimientos de cementicios (CW) se agrupan en el rango de $\mathrm{SRI}=100$ a $80 \%\left(\mathrm{Ts}=35\right.$ a $\left.46^{\circ} \mathrm{C}\right)$. La mayoría de las alternativas de composición cementicia se agrupan en un rango intermedio de SRI entre 60 a $40 \%(\mathrm{Ts}=58$ a $68^{\circ} \mathrm{C}$ ). Estos registros indican que el uso de revestimientos cementicios muestra un comportamiento más ineficiente.

- La terminación Rulato travertino, muestra un mejor desempeño térmico asociado a la terminación fina en todos los colores evaluados. Mientras que en las alternativas Llaneadas los acabados finos o gruesos no muestran una tendencia dominante.

- El color es la variable más importante en los revestimientos acrílicos ya que determina el comportamiento térmico, mientras que para el caso de los revestimientos cementicios se deriva del efecto combinado de textura y color. En el caso de los revestimientos cementicios la selección de color entre las diferentes texturas y terminaciones puede mejorar su comportamiento térmico un $50 \%$ respecto al SRI y disminuir $21^{\circ} \mathrm{C}$ su temperatura superficial (ver CW72-vs. - CW80 en Tabla 3). El color gris plomo están menos influenciados por la terminación y textura, mientras que los colores intermedios (marfil, gris claro, piedra parís, terracota, verde cemento y ocre) tienen diferencias significativas asociadas a estas variables, dónde su temperatura superficial varía entre 12 a $22{ }^{\circ} \mathrm{C}$. Por último, en las tonalidades más claras (blanco, marfil y ocre) el revestimiento acrílico muestra un mejor comportamiento térmico que el cementicio (por ejemplo ver -SIP36 vs. CW76 en Tabla 3) con diferencias de temperatura superficial de $12{ }^{\circ} \mathrm{C}$. Se invierte esta tendencia en la mayoría de los colores que tienen reflectancia solares menores: piedra parís, terracota, gris perla, gris plomo y verde cemento. Es decir que, en estos colores, el revestimiento cementicio tiene un mejor desempeño que el acrílico para disminuir las temperaturas urbanas (por ejemplo ver -CW 80 vs. SIP 40 en Tabla 3). Registrando una diferencia de temperatura superficial de $23{ }^{\circ} \mathrm{C}$. 
Tabla 3. Codificación, denominación, características formales, temperatura superficial ( $\mathrm{Ts}{ }^{\circ} \mathrm{C}$ ) e Índice de reflectancia solar (SRI \%) de materiales evaluados. Cabe destacar, que sólo se describen los materiales que fueron mencionados en el presente trabajo y no el total de los ensayados.

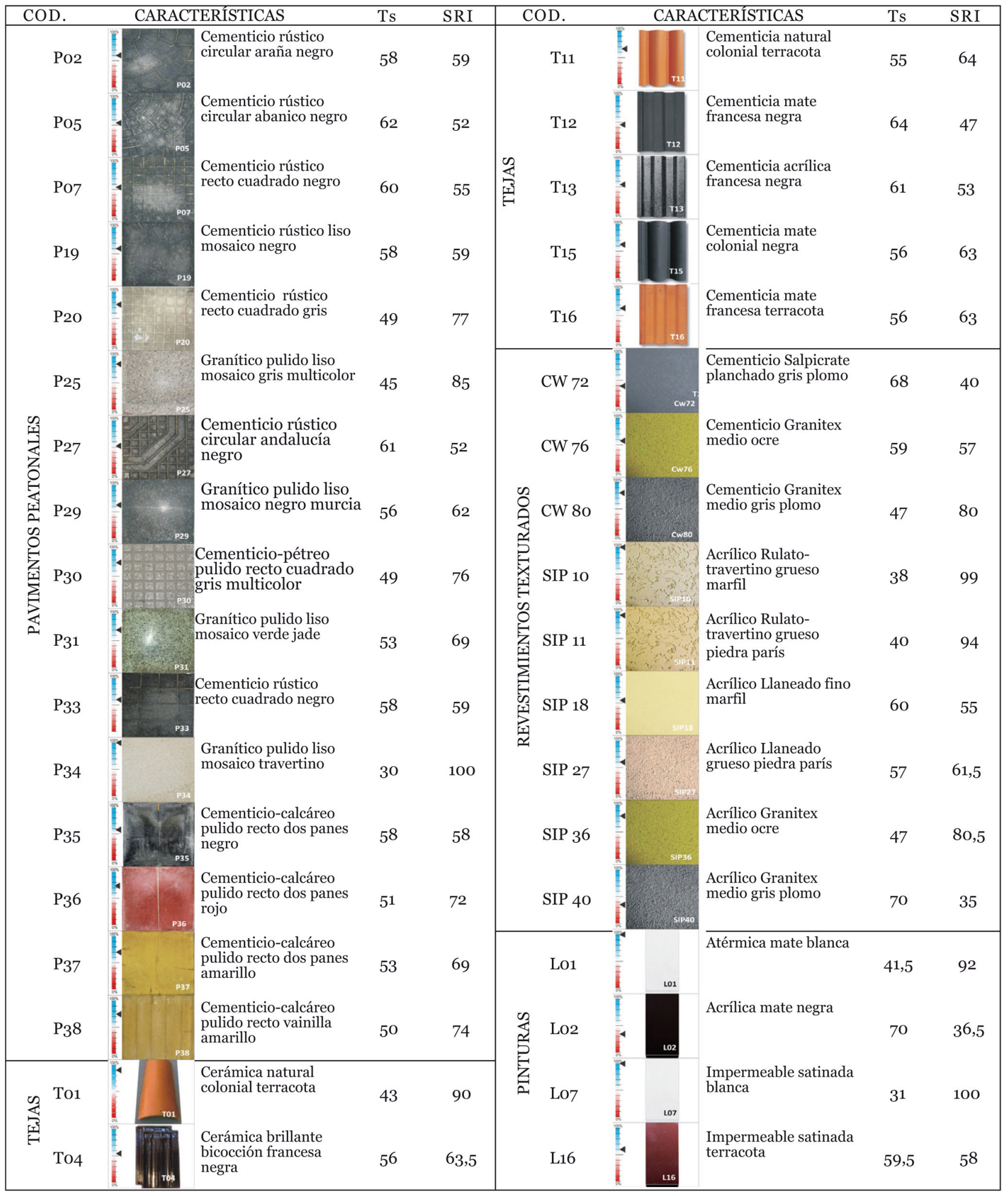

\subsubsection{Pinturas exteriores}

Existe una amplia variedad de pinturas para exterior, es por ello que resulta importante seleccionar la opción adecuada considerando las siguientes variables:

- La composición impermeable es levemente más eficiente que la acrílica, alcanzando una temperatura superficial entre 31 a $43{ }^{\circ} \mathrm{C}$ y SRI entre 100 y $88 \%$. Mientras que la temperatura superficial de las alternativas de composición acrílica se ubica en un rango de $44,5{ }^{\circ} \mathrm{C}$ y $40{ }^{\circ} \mathrm{C}$ y SRI entre 94 a $86 \%$.

Cabe destacar, que la pintura de composición atérmica-Lo1-, a pesar de su condición, no presenta un comportamiento notablemente mejor que el resto de las alternativas, con registros de temperatura superficial de $10,5{ }^{\circ} \mathrm{C}$ por encima de la 
opción más eficiente evaluada (Impermeable satinada blancaL07). Es decir, que no basta con elegir una pintura atérmica, de acuerdo a lo que ofrece el mercado, sino que es necesario un estudio más profundo, que pondere el potencial de enfriamiento pasivo; y de esta forma asegurar que su aplicación en la envolvente edilicia sea efectiva. (Ver Lo1 en Tabla 3).

- El color blanco es el más eficiente, alcanza una temperatura superficial promedio de $41,5{ }^{\circ} \mathrm{C}$ y SRI promedio de $92 \%$ en todas las composiciones. En el caso de las pinturas látex de tonalidades oscuras (terracota y negro), muestran un funcionamiento ineficiente en el orden de reducción de las cargas térmicas de la ciudad. Con una temperatura superficial a $59{ }^{\circ} \mathrm{C}$. (Ver Lo2 y L16 en Tabla 3).

\subsection{Selección de los materiales más resistentes al envejecimiento de acuerdo a sus características}

Para determinar la eficiencia térmica del material es necesario considerar la degradación de la capa superficial del ma- terial debido a las solicitaciones del entorno y acumulación de suciedad que causan modificaciones en sus propiedades ópticas, y por lo tanto, en su aptitud para disminuir las temperaturas superficiales.

Para ello, se analizó la modificación de los niveles de índice de reflectancia solar de los materiales según periodo de exposición y características de clasificación. Inicialmente se observó que en el total de los materiales la distribución de los niveles de SRI1 es dispersa: el $100 \%$ de los pavimentos evaluados, el $94 \%$ de las tejas, el $63 \%$ de los revestimientos y el $88 \%$ de las pinturas registran temperaturas superficiales inferiores a $60{ }^{\circ} \mathrm{C}$ y SRI superior al $50 \%$. Mientras que el drástico desgaste sucede en el tercer período de envejecimiento, dónde las temperaturas superficiales se incrementan entre 12 y $29{ }^{\circ} \mathrm{C}$ y sólo el $30 \%$ de los materiales consigue un SRI3 superior al $50 \%$.

Las temperaturas superficiales del total de los materiales se derivan del efecto combinado de la composición, textura, color y forma, pero el color resulta la variable de mayor impacto

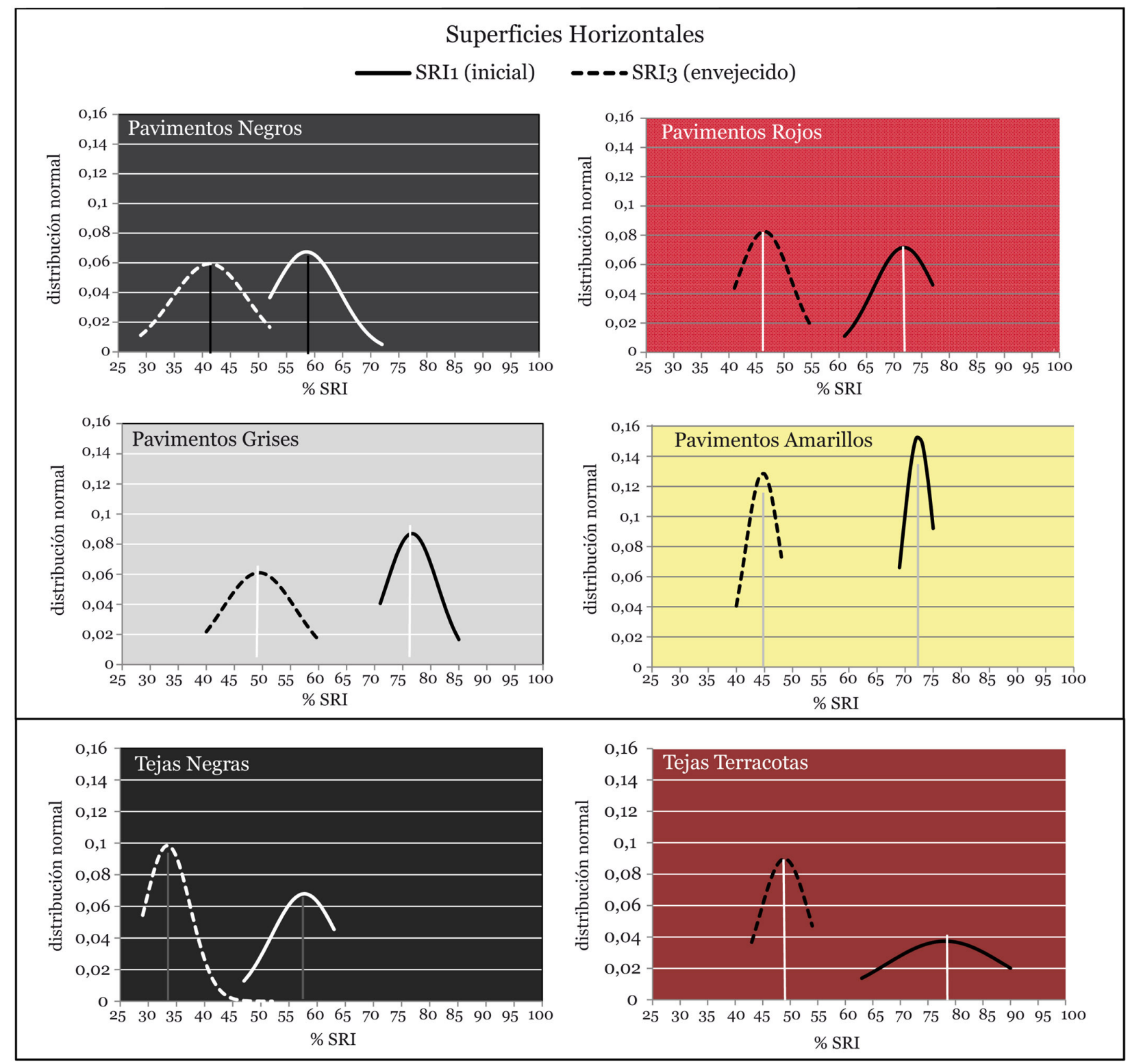

Figura 2. Distribución normal de materiales horizontales -pavimentos y tejas- para la condición inicial (SRI1) y envejecida (SRI3) según color. 




Figura 3. Distribución normal de materiales verticales -revestimientos texturados y pinturas látex- para la condición inicial (SRI1) y envejecida (SRI3) según color. 
en el comportamiento térmico. Es por ello que en la sección 3.3.1 se analiza el efecto del envejecimiento sobre los colores para cada tipología de envolvente (pavimentos, tejas, revestimientos texturados y pinturas). Las Figuras 2 y 3 grafican mediante la función gaussiana la curva normal de niveles de SRI de los materiales bajo la condición nueva (SRI1) y envejecida al tercer año de exposición (SRI3).

\subsubsection{Relación entre color y envejecimiento}

Al comparar los promedios de SRI entre la condición inicial (SRI1) y envejecida (SRI3).de acuerdo a color en los materiales de disposición horizontal (pavimentos y tejas) se observa que las disminuciones de SRI promedio oscilan entre 18 a $29 \%$, es decir que la temperatura superficial se incrementa entre 10 a $18^{\circ} \mathrm{C}$ luego de tres años de exposición (Figura 2).

En las superficies verticales, los revestimientos texturados especialmente en tonalidades claras (blanco, marfil, piedra parís, ocre y gris perla) son las tipologías materiales que menos resisten al envejecimiento, ya que presentan disminuciones de SRI promedio entre 28 a $40 \%$, registrando incrementos de temperatura superficial entre 11 y $18^{\circ} \mathrm{C}$. En contraste con las pinturas blancas que sólo modifican su SRI promedio $11 \%$ y elevan la temperatura superficial $6{ }^{\circ} \mathrm{C}$ luego de envejecidas (Figura 3).

Las gráficas correspondientes a las Figuras 2 y 3 constituyen una herramienta de gran utilidad ya que permiten ponderar las posibilidades que brinda cada color después de su envejecimiento. Por ejemplo, en los pavimentos negros y grises la curtosis de la curva envejecida es mayor que la inicial, lo mismo ocurre en los revestimientos texturados blancos y marfil. Esto significa que las posibilidades de seleccionar alternativas eficientes son mayores para una combinación determinada de color y tipología de envolvente.

Para mostrar la aplicación de esta herramienta a la hora de seleccionar un material adecuado para un determinado proyecto, a modo de ejemplo se contrasta el rendimiento energético de dos soluciones de fachadas -revestimientos texturados blancos en relación a pinturas látex blancas- (Figura 3). Se observa que el SRI inicial promedio de revestimiento texturado blanco es de $94 \%$ y el de las pinturas blancas es de $92 \%$, es decir que ambos materiales registran una temperatura superficial aproximada de $40{ }^{\circ} \mathrm{C}$. Sin embargo durante la etapa de envejecimiento el SRI promedio al final del tercer periodo es mucho menor en los revestimientos que en las pinturas (SRI3 revestimiento $=54 \% v s$. SRI3 pintura $=81 \%$ ). En este periodo el revestimiento texturado eleva $12{ }^{\circ} \mathrm{C}$ su temperatura superficial sobre las pinturas. Este hecho pone de manifiesto que si se desea seleccionar el color blanco para la resolución de una fachada, las pinturas brindan mejores prestaciones térmicas, permaneciendo más fresca luego de su envejecimiento. (Ver Revestimientos Blancos vs. Pinturas Blancas en Figura 3).

\section{RESULTADOS A ESCALA URBANA}

Entre otros aspectos, la sustentabilidad de las ciudades se apoya en el uso racional de los recursos energéticos y la preservación de los recursos ambientales, en este sentido las temperaturas del aire y de las superficies urbanas, condicionan tanto el consumo energético de los edificios, como la habitabilidad de los espacios exteriores. Es por ello importante valorar el efecto de la selección de la envolvente sobre el comportamiento de un recinto urbano.

\subsection{Efecto de la selección de materiales sobre la eficiencia térmica urbana}

Los resultados obtenidos mediante la simulación con ENVImet de los 6 escenarios propuestos se detallan en la Tabla 4.

Si analizamos los datos de temperatura de aire del total de los escenarios se observa que en baja densidad aumentar los niveles reflectancia solar (R2) resulta una estrategia beneficiosa en torno a disminuir las temperaturas máximas y promedio de aire. En cambio, en la alta densidad esta estrategia resulta perjudicial durante el período de calentamiento ( $17 \mathrm{hs})$, debido a que se disminuye la porción de visión efectiva al cielo dado por la altura edilicia quedando atrapada la radiación reflejada por las superficies con alto reflectancia solar. Estas múltiples reflexiones dentro del cañón vial aumentan la temperatura de las superficies y del aire circundante como consecuencia del incremento de la temperatura media radiante.

Tanto para la baja, como para la alta densidad los escenarios con reflectancia solar combinada ( $\mathrm{R}_{3}$-alta reflectancia solar en superficies horizontales y baja reflectancia solar en superficies verticales-), registran menor temperatura de aire para todas las configuraciones ensayadas.

$\mathrm{Al}$ analizar los resultados de temperatura del modelo de suelo de ENVI-met evidencia que la temperatura mínima de suelo coincide con el horario de registro de las temperatura mínimas de aire ( $8 \mathrm{hs}$ ). Sin embargo durante las máximas temperaturas esto no sucede. La temperatura de suelo máxima ocurre a las $16 \mathrm{hs}$, y la temperatura máxima de aire es a las 17 hs, es decir que se registra una inercia térmica de una hora.

La modificación de las temperaturas superficiales según estrategia es mucho mayor que las producidas sobre las temperaturas de aire. Mientras que a las 17 hs las temperaturas máximas de aire según escenario oscilan entre 32 a $36{ }^{\circ} \mathrm{C}$, las temperaturas superficiales de suelo oscilan entre 40 y $52{ }^{\circ} \mathrm{C}$.

Se observa que el caso actual de Mendoza, con baja densidad y reflectancia solar baja (R1) es una de las alternativas que mayor temperatura de suelo registra, hecho que pone en evidencia la importancia de incrementar los niveles de reflectancia solar de las superficies horizontales para mejorar la habitabilidad de los espacios exteriores.

Tabla 4. Temperaturas de aire $\left(\mathrm{C}^{\circ}\right)$ y suelo $\left(\mathrm{C}^{\circ}\right)$ mínimas (MIN), máximas (MAX), promedio (PROM) y amplitud (AMP), según densidad edilicia y rango de reflectancia solar de la materialidad de la envolvente (R1: albedo bajo; R2: albedo alto; R3: albedo combinado).

\begin{tabular}{|c|c|c|c|c|c|c|c|}
\hline \multirow{2}{*}{\multicolumn{2}{|c|}{ TEMPERATURAS ${ }^{\circ} \mathrm{C}$}} & \multicolumn{3}{|c|}{ Baja Densidad } & \multicolumn{3}{|c|}{ Alta Densidad } \\
\hline & & \multirow{2}{*}{\begin{tabular}{|c|}
$\mathbf{R 1}$ \\
22 \\
\end{tabular}} & \multirow{2}{*}{$\begin{array}{r}\text { R2 } \\
22 \\
\end{array}$} & \multirow{2}{*}{\begin{tabular}{|r|}
$\mathbf{R}_{3}$ \\
22 \\
\end{tabular}} & \multirow{2}{*}{\begin{tabular}{|r|}
$\mathbf{R 1}$ \\
23 \\
\end{tabular}} & \multirow{2}{*}{$\begin{array}{l}\text { R2 } \\
23.5 \\
\end{array}$} & \multirow{2}{*}{$\begin{array}{r}\mathbf{R 3} \\
23 \\
\end{array}$} \\
\hline \multirow{4}{*}{ 窖 } & MIN (8 hs) & & & & & & \\
\hline & $\operatorname{MAX}(17 \mathrm{hs})$ & 36 & 34 & 33 & 34 & 35 & 32 \\
\hline & PROM & 28 & 27 & 27 & 28 & 28 & 27 \\
\hline & AMP & 3 & 2 & 12 & 11 & 11 & 1 \\
\hline \multirow{4}{*}{$\begin{array}{l}0 \\
\text { 空 } \\
\text { ? }\end{array}$} & MIN (8 hs) & 27 & 26 & 26 & 28 & 28 & 27 \\
\hline & MAX (16 hs) & 51 & 43 & 40 & $5^{2}$ & 48 & 40 \\
\hline & PROM & 37,5 & 34 & 32 & 38 & 39 & 33 \\
\hline & AMP & 24 & 26 & 26 & 28 & 28 & 27 \\
\hline
\end{tabular}


Basándonos en los resultados previamente obtenidos, se observa que la estrategia de incrementar la reflectancia solar en las envolventes registra un efecto térmico benéfico sobre los materiales horizontales (pavimentos y techos) dado a su posición relativa con respecto al cielo que favorece el enfriamiento radiativo, en cambio el aumento de reflectancia solar en las superficies verticales produce un sobrecalentamiento debido a las múltiples reflexiones entre fachadas.

\section{DISCUSIÓN Y CONCLUSIONES}

Este estudio demuestra que la adecuada selección de los materiales que componen la envolvente urbana es una estrategia costo-efectiva, ya que contribuye al enfriamiento pasivo de las ciudades y no requiere prácticamente ningún costo adicional para su aplicación, solo es necesario disponer de información acerca del comportamiento térmico del material.

De modo particular el color es la variable que resulta más impactante en el comportamiento térmico superficial de un material, registrando diferencias de temperatura superficial de 20 a $40{ }^{\circ} \mathrm{C}$ entre casos extremos (ver L07 vs. Lo2 y P29 vs. P34 en Tabla 3). Sin embargo se observaron otras variables relevantes que determinan la aptitud de un material, como la terminación y composición. Seleccionar una textura (ver SIP10 vs. SIP18 en Tabla 3) o una composición (ver SIP40 vs. CW8o y To1 vs. T11 en Tabla 3) adecuada manteniendo el resto de las características constantes puede mejorar el desempeño térmico de un material entre 10 y $20{ }^{\circ} \mathrm{C}$. Es decir que se puede mejorar el desempeño térmico del espacio urbano o el edificio sin sacrificar la estética en términos de su color, utilizando una combinación eficiente de diferentes características del material.

Otra variable que debe ser considerada es la resistencia de los materiales al envejecimiento. La capacidad reflectiva (albedo) de los materiales disminuye considerablemente con el paso del tiempo. Sólo el $30 \%$ de los materiales consigue un SRI superior al 50 \% luego de 3 años de exposición, siendo la pintura la tipología de material más resistente al envejecimiento.

En función de las prestaciones que ofrece el SRI como indicador del desempeño térmico de los materiales durante toda su vida útil, es posible establecer el período en que el material debe ser rehabilitado o reemplazado. El SRI fue seleccionado debido a que permite homologar a nivel internacional los resultados del funcionamiento de los materiales utilizados a nivel local y regional, y al mismo tiempo generar conocimiento en torno al etiquetado energético de los materiales sentando las bases para propiciar una futura certificación energética tanto a nivel urbano como edilicio.

A escala urbana, la aplicación de esta estrategia de enfriamiento permitiría reducir hasta $3{ }^{\circ} \mathrm{C}$ la temperatura del aire y $12{ }^{\circ} \mathrm{C}$ la temperatura de las superficies en la ciudad.

Los resultados obtenidos en este trabajo corroboran la información aportada por artículos científicos producidos previamente. Sin embargo entre sus aportes podemos señalar:

- Demuestra que existen otras características además del color de un material que influyen sobre su comportamiento reflectivo. -Cuantifica la capacidad reflectiva de los mate- riales opacos mediante un índice normalizado como es el SRI, lo que permite la universalización de los resultados. -Pone en evidencia que como el acabado de un material opaco varía con el envejecimiento, sus propiedades reflectivas serán dinámicas en el tiempo y por lo tanto evalúa y cuantifica cómo se afecta la medida del SRI con el envejecimiento.

- Existe, como se expresó anteriormente, gran cantidad de información de carácter técnico que avala la selección adecuada de los materiales como una técnica efectiva para el enfriamiento pasivo de las ciudades, sin embargo, pocos discuten de qué manera esta información puede ser transferida a los responsables de la producción del hábitat. En este sentido, el trabajo aporta información concreta sobre el comportamiento de los materiales opacos disponibles en el mercado local y discute cómo su comportamiento es afectado por el envejecimiento. A mayor escala los aspectos observados podrán ser tenidos en cuenta, para establecer qué información es necesaria para el etiquetado energético de este tipo de productos.

- Haciendo uso de modelos teóricos de alta complejidad, cuantifica el impacto de modificar las propiedades ópticas de las envolventes sobre las temperaturas de aire y suelo en distintos escenarios urbanos de la ciudad analizada.-Finalmente, a partir de combinar los resultados de las simulaciones y la caracterización de los materiales, detecta que los materiales disponibles a escala local para la resolución de las superficies horizontales de la ciudad son los que presentan peor performance y dada su importancia en la determinación de las temperaturas de la ciudad, se concluye que la industria local debe poner énfasis en el desarrollo de nuevos productos.

\section{NOMENCLATURAS}

â Reflectancia solar o albedo

AMM Área metropolitana de Mendoza

CW Revestimientos texturados cementicios

$\mathrm{H} \quad$ Altura edilicia promedio

he Coeficiente convectivo (Wm-2K-1).

(Valor adoptado de hc = 6,4 Wm-2K-1).

ICU Isla de calor urbana

L Pinturas de exterior

P Pavimentos peatonales

R1 Escenario rango 1 (â bajo)

R2 Escenario rango 2 (â alto)

R3 Escenario rango 3 (â combinado)

SIP Revestimientos texturados acrílicos

SRI Índice de reflectancia solar (\%)

T Tejas

$\mathrm{Tb}$ Temperatura superficial del patrón negro $\left({ }^{\circ} \mathrm{K}\right)$

Ts Temperatura superficial $\left({ }^{\circ} \mathrm{C}\right)$

Tw Temperatura superficial del patrón blanco $\left({ }^{\circ} \mathrm{K}\right)$

W Ancho de canal vial

$\alpha \quad$ Absortancia solar $=1-\hat{a}$

$\varepsilon \quad$ Emisividad

\section{AGRADECIMIENTOS}

A la Agencia Nacional de Promoción Científica y Tecnológica -ANPCYT- y al Consejo Nacional de Investigaciones Científicas y Técnicas -CONICET- que proporcionaron los fondos para la presente investigación. 


\section{REFERENCIAS}

(1) Millennium Ecosystem Assessment. (2005). Ecosystems and Human Well-being e Current State and Trends. Washington, DC: Island Press.

(2) Madlener, R., Sunak, Y. (2011). Impacts of urbanization on urban structures and energy demand: What can we learn for urban energy planning and urbanization management. Sustainable Cities and Society, 1(1): 45-53, doi: http://dx.doi. org/10.1016/j.scs.2010.08.006.

(3) International Energy Agency (IEA). (2013). Key World Energy Statistic. http://www.iea.org/publications/freepublications/publication/KeyWorld2013.pdf.

(4) Perez-Lombard, L., Ortiz, J., Pout, C. (2008). A review on buildings energy consumption information. Energy Building, 40(3): 394-398, doi: http://dx.doi.org/10.1016/j.enbuild.2007.03.007.

(5) Santamouris, M. (2007). Heat island research in Europe - The state of the art. Journal Advances Building Energy Research, 1(1): 123-150, doi: http://dx.doi.org/10.1080/17512549.2007.9687272.

(6) Mirzaei, P., Haghighat, F. (2010). Approaches to study urban heat island - Abilities and limitations. Building and Environment, 45(10): 2192-2201, doi: http://dx.doi.org/10.1016/j.buildenv.2010.04.001.

(7) Founda, D. (2011). Evolution of the air temperature in Athens and evidence of climatic change: A review. Advances in Building Energy Research, 5(1): 7-41, doi: http://dx.doi.org/10.1080/17512549.2011.582338.

(8) Mihalakakou, P., Santamouris, M., Papanikolaou, N., Cartalis, C., Tsangrassoulis, A. (2004). Simulation of the urban heat island phenomenon in Mediterranean Climates. Journal of Pure and Applied Geophysics, 161(2): 429-451, doi: http://dx.doi.org/10.1007/sooo24-003-2447-4.

(9) Livada, I., Santamouris, M., Niachou, K., Papanikolaou, N., Mihalakakou, G. (2002). Determination of places in the Great Athens Area where the Heat Island Effect is Observed. Theoretical and Applied Climatology, 71(3-4): 219-230, doi: http://dx.doi.org/10.1007/s007040200006.

(10) Mohsin, T., Gough, W. (2012). Characterization and estimation of urban heat island at Toronto: Impact of the choice of rural sites. Theoretical and Applied Climatology, 108(1-2): 105-117, doi: http://dx.doi.org/10.1007/s00704-011-0516-7.

(11) Papanastasiou, D., Kittas, C. (2012). Maximum urban heat island intensity in a medium-sized coastal mediterranean city. Theoretical and Applied Climatology, 107(3-4): 407-416, doi: http://dx.doi.org/10.1007/s00704-011-0491-z.

(12) ASTM. (2011). ASTM E1980-11. Standard practice for calculating solar reflectance index of horizontal and low-sloped opaque surfaces. ASTM International.

(13) Bruse, M. (1999). Die Auswirkungen kleinskaliger Umweltgestaltung auf das Mikroklima. Entwicklung des prognostischen numerischen Modells ENVI-met zur Simulation der Wind-, Temperatur, und Feuchtverteilung in städtischen Strukturen (PhD. Thesis). Germany: Univ. Bochum.

(14) Krüger, E., Minella, F., Rasia, F. (2011). Impact of urban geometry on outdoor thermal comfort and air quality from field measurements in Curitiba, Brazil. Building and Environment, 46(3): 621-634, doi: http://dx.doi.org/10.1016/j.buildenv.2010.09.006.

(15) Soo-Gon, H., Sun-Hye, M., Jung-Ho, H. (2007). Changes of the micro-climate and building cooling load due to the green effect of a restored stream in Seoul, Korea. En Proceedings Building Simulation (pp. 1131-1138).

(16) Samaali, M., Courault, D., Bruse, M., Olioso, A., Occelli, R. (2007). Analysis of a 3D boundary layer model at local scale: Validation on soybean surface radiative measurements. Atmospheric Research, 85(2): 183-198, doi: http://dx.doi. org/10.1016/j.atmosres.2006.12.005.

(17) Bruse, M. (2009). Stadtlandschaft im Klimawandel-Zu den Grundlagen des städtischen Mikroklimas. En Collinet, H.D., Pesch, F. (Eds.) Stadt und Landschaft. Essen: Klartext Verlag.

(18) Chowa, W., Brazel, A. (2012). Assessing xeriscaping as a sustainable heat island mitigation approach for a desert city. Building and Environment, 47: 170-181, doi: http://dx.doi.org/10.1016/j.buildenv.2011.07.027.

(19) Perini, K., Magliocco, A. (2014). Effects of vegetation, urban density, building height, and atmospheric conditions on local temperatures and thermal comfort. Urban Forestry and Urban Greening, 13(3): 495-506, doi: http://dx.doi. org/10.1016/j.ufug.2014.03.003.

(20) Taleghani, M., Kleerekoper, M., Tenpierik, M., Dobbelsteen, A. (2014). Outdoor thermal comfort within five different urban forms in the Netherlands. Building and Environment, 83: 65-78, doi: http://dx.doi.org/10.1016/j.buildenv.2014.03.014.

(21) Ali-Toudert, F. (2005). Dependence of outdoor thermal comfort on street design in hot and dry climate. Berichte des Meteorologischen Institutes der Universität Freiburg No. 15. http://www.freidok.uni-freiburg.de/volltexte/2078.

(22) ASTM. (2006). ASTM E1933-99a: American Society for Testing and Materials. Standard Test Methodos for measuring and compensating for emissivity using infrared imaging radiometers. ASTM International.

(23) Akbari, H., Levinson, R., Stern, S. Procedure for measuring the solar reflectance of flat or curved roofing assemblies. Solar Energy, 82(7): 648-655, doi: http://dx.doi.org/10.1016/j.solener.2008.01.001.

(24) Correa, E., Ruiz, M. A., Canton, A., Lesino, G. (2012). Thermal comfort in forested urban canyons of low building density. An assessment for the city of Mendoza, Argentina. Build and Environment, 58: 219-230, doi: http://dx.doi. org/10.1016/j.buildenv.2012.06.007. 